stress and DNA methylation. The mechanism of SCE induction, which is now generally believed to involve the replication fork, and its modulation through DNA repair, potentiating agents, and anticlastogens is also investigated. Characterisation of SCEs and the concomitant chromosomal aberrations is attempted and the interrelationship of SCEs, clastogenesis, mutagenesis, cracinogenesis, and environmental pollutants is examined in some detail. This volume concludes with papers on the statistical treatment of the subject.

Part B. Genetic Toxicology and Human Studies first addresses the use of SCE studies in investigating the effects of mutagens in vertebrate systems, both in vitro and in vivo; SCE persistence and the influence of cell cycle kinetics are examined in the human lymphocyte culture system in particular. The value of SCE studies in the diagnosis of inherited genetic disease and in the dosimetry of acquired genetic damage resulting from the use of various pharmaceutical products, especially cytostatic drugs, and environmental and occupational agents, such as cigarette smoke and styrene, is assessed: in this way the possibly premature concept of biomonitoring using SCE levels is tentatively explored. In addition, the inherent variability of spontaneous and induced SCE levels observed in man is evaluated as a possible factor in predisposition to cancer. The treatise ends with the summary of a general and apparently inconclusive discussion of the interpretation and predictive value of SCE data and its relevance to public health.

The fascinating phenomenon of SCE, despite recent advances, remains enigmatic with both its precise mechanism of induction and its clinical significance yet to be fully elucidated. Nevertheless, this treatise is a timely and very informative publication, which, considering the limitations imposed by the content, is quite well presented, the main deficiency being the lack of any meaningful synthesis of the mass of data reported. Overall, it is an excellent reference book and provides stimulating reading for researchers and those with a keen interest in cytogenetics, genetic toxicology, and related specialties.

\section{Atlas of Skeletal Dysplasias}

By R Wynne-Davies, C M Hall, and A G Apley. (Pp 600; figures + tables. $£ 90.00$.) Edinburgh: Churchill Livingstone. 1985.

This book is not the first to be devoted to bone dysplasias, but it is certainly quite different from any other that exists and stands in a class of its own. As its name implies, it is an atlas, but it is much more than this, being rather a detailed account of bone dysplasias as seen principally through their radiographic appearance.

This book starts with some useful introductory sections on normal $x$-ray appearances and prenatal detection, after which the major groups of primary bone dysplasias are dealt with successively and in detail. Wisely, the authors do not attempt to cover general malformations or limb defects except as they may affect bone dysplasias.

It is in the detailed treatment of the individual disorders that the book excels; $x$-rays are given in abundance to illustrate different manifestations at different ages, giving a much fuller and more balanced picture than is possible when only a few selected illustrations are allowed. Introducing each series of films is a brief but comprehensive text, which is particularly strong on natural history and complications, and almost always accurate regarding inheritance.

Inevitably in a volume of over 600 large pages, some faults can be found, but most are trivial; on genetics the only error I could find was the one widely repeated elsewhere of lethal osteogenesis imperfecta being recessively inherited. My only general criticism is that the standard of clinical photography is not always as high as that of the $x$-rays; care of background could have greatly improved some of these. The lack of gonadal shielding in a high proportion of $x$-rays is an indictment of much orthopaedic and radiological practice, but cannot be blamed on the authors!

Leaving aside these selected points the book as a whole is an outstanding achievement. It is thoroughly enjoyable to read, and is quite clearly the work of authors who are familiar with and experts on bone dysplasias as a whole, not just on their radiology or any other single aspect. It is this integrated approach that will give the book its real and lasting value. Despite its price it will surely prove an essential companion to anyone involved in the clinical and genetic management of this fascinating group of disorders. 